\title{
Influence de la congélation et de la cryo-dessiccation sur le taux de survie et le pourcentage des ferments lactiques dans le yaourt
}

\author{
par \\ A. JABARIT \\ SOCALTRA - Centre de Recherches et d'Essais \\ 128, boulevard Victor-Hugo, 92 - Clichy (France)
}

\section{INTRODUCTION}

Au cours de ces dernières années, SOCALTRA, Centre de Recherche et de développement dans le domaine des Industries Agricoles et Alimentaires, 128, boulevard Victor-Hugo à Clichy, s'est spécialisée dans la cryo-dessiccation des produits alimentaires aussi bien que des produits pharmaceutiques et diététiques.

C'est ainsi que dans le courant de l'année 1968, la Délégation Générale à la Recherche Scientifique et Technique, nous a permis d'entreprendre, dans le cadre d'une action concertée, une étude sur la sélection des souches des ferments lactiques, du yaourt destiné à la cryo-dessiccation.

Notre but est d'étudier l'influence de la congélation sur la survie et le comportement des ferments lactiques du yaourt, en appliquant différentes températures basses sur un certain nombre déterminé de souches des ferments lactiques, tels que :

- Lactobacillus bulgaricus,

- Streptococcus thermophilus.

Nous étudions également la survie et la viabilité des ferments lactiques pendant la cryo-dessiccation ainsi que leur revivification au cours du vieillissement de ce produit (stockage de longue durée).

Dans ce fascicule, nous présentons une partie de nos études concernant l'influence de la congélation et de la cryo-dessiccation sur le taux de survie et le pourcentage des ferments lactiques (Streptococcus thermophilus et Lactobacillus bulgaricus) dans le yaourt. 
Une étude ultérieure concernera d'autres ferments ci-dessous mentionnés :

- Streptococcus lactis,

- Lactobacillus acidophilus,

- Lactobacillus helveticus.

\section{PROTOCOLE D'ESSAI}

\section{Première partie} vant :

Le protocole utilisé pour un essai est schématiquement le sui-

- Préparer et stériliser du lait destiné à la fabrication du yaourt dont la composition est la suivante :

- lait écrémé en poudre : $140 \mathrm{~g}$,

- eau distillée Q.S.P. : $1000 \mathrm{ml}$.

- Ensemencer à 5 p. 100 avec du levain connu de nos ferments lactiques de laboratoire:

- Streptococcus thermophilus,

- Lactobacillus bulgaricus.

- Etuver à $43^{\circ} \mathrm{C}$ pendant deux heures.

- Laisser refroidir progressivement le yaourt afin d'éviter le choc thermique, auquel les ferments lactiques sont excessivement sensibles et laisser continuer la fermentation lentement, jusqu'à la phase stationnaire; à ce stade la viabilité des ferments lactiques est maximale (1).

- Prélever et contrôler :

- la viabilité : nombre des ferments lactiques revivifiables par ml (5),

- le pourcentage des ferments lactiques,

- l'acidité en degré Dornic,

- le $\mathrm{pH}$.

\section{Congélation et conservation}

En rapport avec notre série d'expériences, contrôles systématiques identiques à ceux du yaourt frais, le temps de congélation aux températures basses $\left(-20^{\circ},-25^{\circ},-30^{\circ},-35^{\circ},-40^{\circ}\right)$, varie entre 1 h $15 \mathrm{mn}$, et 2 h 45 mn (graphique $\mathrm{n}^{\circ} 3$ ).

La durée de congélation et de conservation pour chaque température est de 15 heures, c'est-à-dire toute la nuit de $18 \mathrm{~h}$ à $9 \mathrm{~h}$ le lendemain matin. 


\section{Détermination de la charge optimale}

D'après nos expériences, compte tenu de la durée du travail jour nalier, nous avons choisi une charge de $1,800 \mathrm{~kg}$, soit $8 \mathrm{~kg} / \mathrm{m}^{2}$.

\section{Durée du cycle}

La durée du cycle de la cryo-dessiccation varie entre 8 et 9 heures, ce qui correspond à la durée d'une journée de travail.

\section{Cryo-dessiccation du yaourt}

La sublimation du yaourt s'effectue de façon différente suivant la nature de l'eau contenue dans le yaourt.

Dans le yaourt, l'eau est répartie sous différentes formes (7)

- eau liée ou eau d'hydratation des protéines,

- eau immobilisée ou eau capillaire,

- eau libre ou mobile en sérum,

- eau vitale des ferments lactiques et eau non cristallisable des complexes lipido-protidiques.

Voici les différentes phases de sublimation :

a) Première phase : l'eau de sérum et l'eau capillaire (dessiccation primaire);

b) deuxième phase : eau liée ou eau d'hydratation des protéines (dessiccation secondaire);

c) troisième phase : eau vitale des ferments lactiques et eau non cristallisable des complexes lipido-protidiques (dessiccation tertiaire) (10).

\section{Détermination de la fin de la sublimation ou de l'humidité résiduelle}

Cette étape du processus de la cryo-dessiccation est extrêmement importante, en particulier sur la viabilité des ferments lactiques et leur survie au cours du vieillissement.

A la fin de la dessiccation primaire, le pourcentage de l'humidité résiduelle est supérieure à 8 p. 100 . Au cours de la dessiccation secondaire l'humidité résiduelle peut varier entre 4 et 7 p. 100. Pendant la dessiccation tertiaire, son pourcentage se situe entre 4 et 1 p. 100 (voire au-dessous de 1 p. 100) ce qui risque de provoquer la réaction de Maillard (3 et 6).

Vers la fin du cycle, l'écart d'échange thermique est faible, les températures basses (le yaourt) et les températures hautes (plaques chauffantes) se stabilisent à la température de consigne, fixée à l'avance sur la surface du yaourt $\left(35^{\circ} \mathrm{C}\right)$. 
Le yaourt cryo-desséché est mis en emballage sous-vide et compensé à l'azote.

\section{Prélèvements et analyses du yaourt cryo-desséché}

Le yaourt cryo-desséché est soumis aux contrôles suivants :

- analyse physico-chimique,

- détermination de l'humidité résiduelle (4 et 9),

- analyses biochimiques et bactériologiques (5),

- analyses sensorielles et organoleptiques,

- test de reconstitution par hydratation,

- dégustation et appréciation, selon les méthodes subjectives et objectives.

\section{Deuxième partie : Résultats expérimentaux}

Nous étudions les résultats obtenus pour les expériences concernant l'influence des températures basses sur la viabilité et le pourcentage des ferments lactiques au cours de la congélation et de la cryo-dessiccation qui s'ensuit. Nous les présentons sous la forme de deux tableaux récapitulatifs $\mathrm{n}^{\circ} 1$ et $\mathrm{n}^{\circ} 2$.

Le graphique A montre la pression de vapeur au cours de la cryo-dessiccation et sa relation directe avec la température du produit à lyophiliser. Il serait donc souhaitable de déterminer la pression de vapeur pour chaque produit au cours de la cryo-dessiccation.

Les graphiques $n^{\circ} 1$ et $n^{\circ} 2$ nous permettent d'évaluer la perte que subissent les ferments lactiques au cours de la congélation en particulier les lactobacillus bulgaricus.

Sur le graphique $n^{\circ} 3$, nous pouvons constater que le temps de congélation, aux différentes températures basses pour une quantité bien déterminée de yaourt $(1800 \mathrm{~g})$ varie entre $1 \mathrm{~h} 15 \mathrm{mn}$ et $2 \mathrm{~h} 45 \mathrm{mn}$, ce qui représente une vitesse de congélation de 3 à $8 \mathrm{~mm}$ à l'heure.

D'autre part, dans le graphique $\mathrm{n}^{\circ} 4$, nous avons présenté des exemples de cryo-dessiccation proprement dite avec ses différentes phases (dessiccations primaire, secondaire et tertiaire).

Nous avons constaté que la dessiccation tertiaire est très importante sur le pourcentage de l'humidité résiduelle et sur la qualité du produit cryo-desséché (yaourt).

Enfin, les graphiques no 5 et 6 nous montrent le point eutectique du yaourt sous l'influence des températures basses sur le pourcentage de l'eau cristallisable dans le yaourt. 
TABLEAU RECAPITULATIF $N^{\circ} 1$

Nombre des ferments lactiques revivifiables dans les yaourts : frais, congelés et lyophilisés (par ml)

\begin{tabular}{|c|c|c|c|c|c|c|}
\hline \multirow{2}{*}{ Yaourt } & \multicolumn{5}{|c|}{ Température de congélation } & \multirow{2}{*}{ Observations } \\
\hline & $-20^{\circ}$ & $-25^{\circ}$ & $-30^{\circ}$ & $-35^{\circ}$ & $-40^{\circ}$ & \\
\hline Frais & $10^{12}$ & $10^{12}$ & $10^{12}$ & $10^{12}$ & $10^{12}$ & \\
\hline Congelé ... & $10^{11}$ & $10^{11}$ & $10^{10}$ & $10^{9}$ & $10^{9}$ & \\
\hline Lyophilisé & $10^{10}$ & $10^{10}$ & $10^{9}$ & $10^{9}$ & $10^{9}$ & \\
\hline
\end{tabular}

On constate que les températures basses à partir de $-30^{\circ}$ ont une influence notable sur le taux de survie des ferments lactiques 
TABLEAU RECAPITULATIF No 2

Pourcentage des ferments lactiques dans les yaourts : frais, congelés et lyophilisés

\begin{tabular}{|c|c|c|c|c|c|c|c|}
\hline \multirow{2}{*}{\multicolumn{2}{|c|}{ Yaourt }} & \multicolumn{5}{|c|}{ Température de congélation } & \multirow{2}{*}{ Observations } \\
\hline & & $-20^{\circ}$ & $-25^{\circ}$ & $-30^{\circ}$ & $-35^{\circ}$ & $-40^{\circ}$ & \\
\hline 㺃 & $\begin{array}{l}\mathrm{S} \\
\mathrm{L}\end{array}$ & $\begin{array}{l}52 \\
48\end{array}$ & $\begin{array}{l}51 \\
49\end{array}$ & $\begin{array}{l}50 \\
50\end{array}$ & $\begin{array}{l}51 \\
49\end{array}$ & $\begin{array}{l}50 \\
50\end{array}$ & \\
\hline $\begin{array}{l}\frac{U}{\mathbb{d}} \\
\text { ơ } \\
0 \\
0\end{array}$ & $\begin{array}{l}\mathrm{S} \\
\mathrm{L}\end{array}$ & $\begin{array}{l}58 \\
42\end{array}$ & $\begin{array}{l}60 \\
40\end{array}$ & $\begin{array}{l}55 \\
45\end{array}$ & $\begin{array}{l}54 \\
46\end{array}$ & $\begin{array}{l}53 \\
47\end{array}$ & \\
\hline 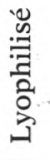 & $\begin{array}{l}\mathbf{S} \\
\mathrm{L}\end{array}$ & $\begin{array}{l}57 \\
43\end{array}$ & $\begin{array}{l}58 \\
42\end{array}$ & $\begin{array}{l}54 \\
46\end{array}$ & $\begin{array}{l}54 \\
46\end{array}$ & $\begin{array}{l}54 \\
46\end{array}$ & \\
\hline
\end{tabular}

On constate que les températures basses ont un effet nuisible sur la survie des lactobacillus bulgaricus, ce qui provoque la disproportion entre les ferments lactiques 


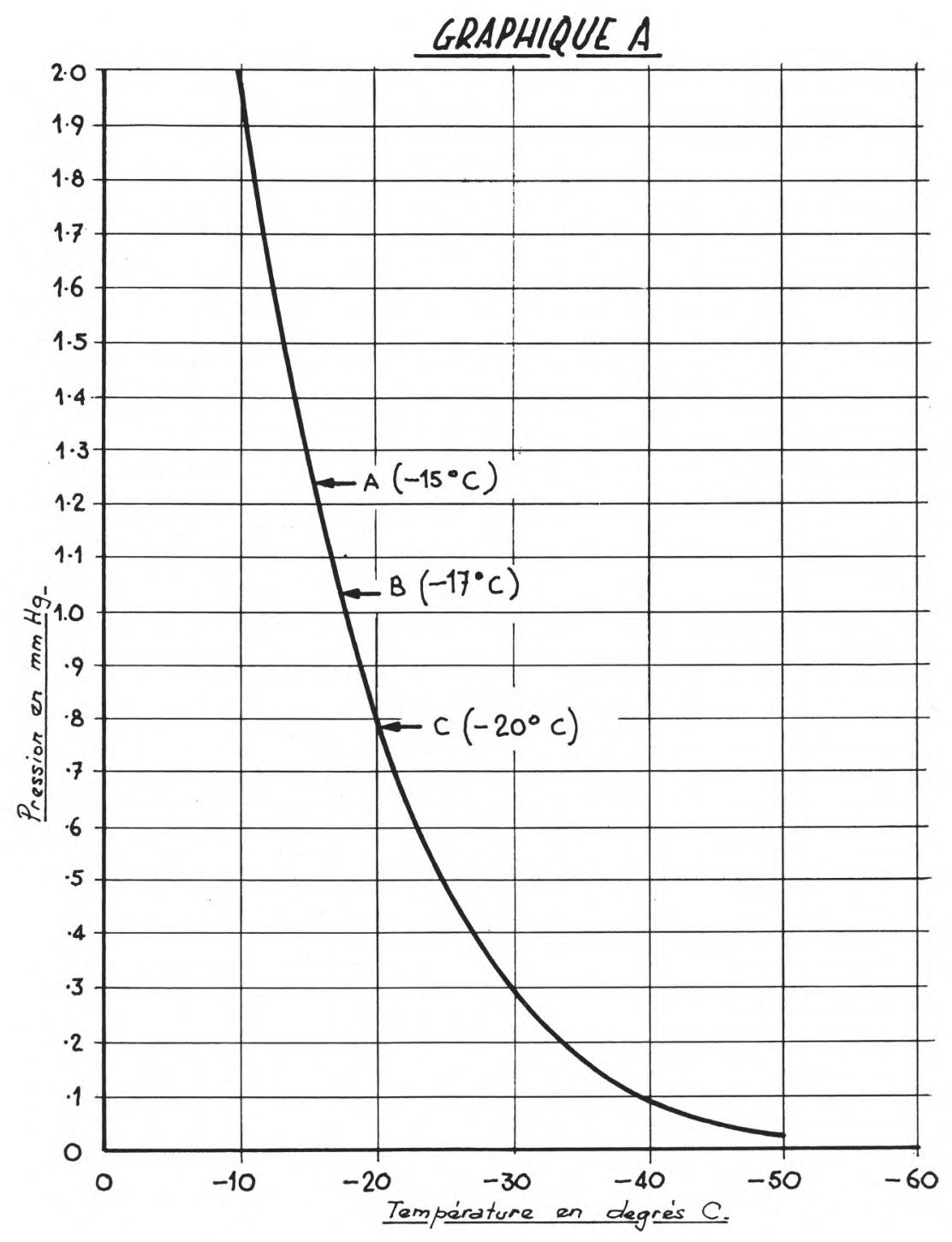

Pression de la vapeur de glace distillée aux différentes températures basses au cours de la cryo-dessiccation 


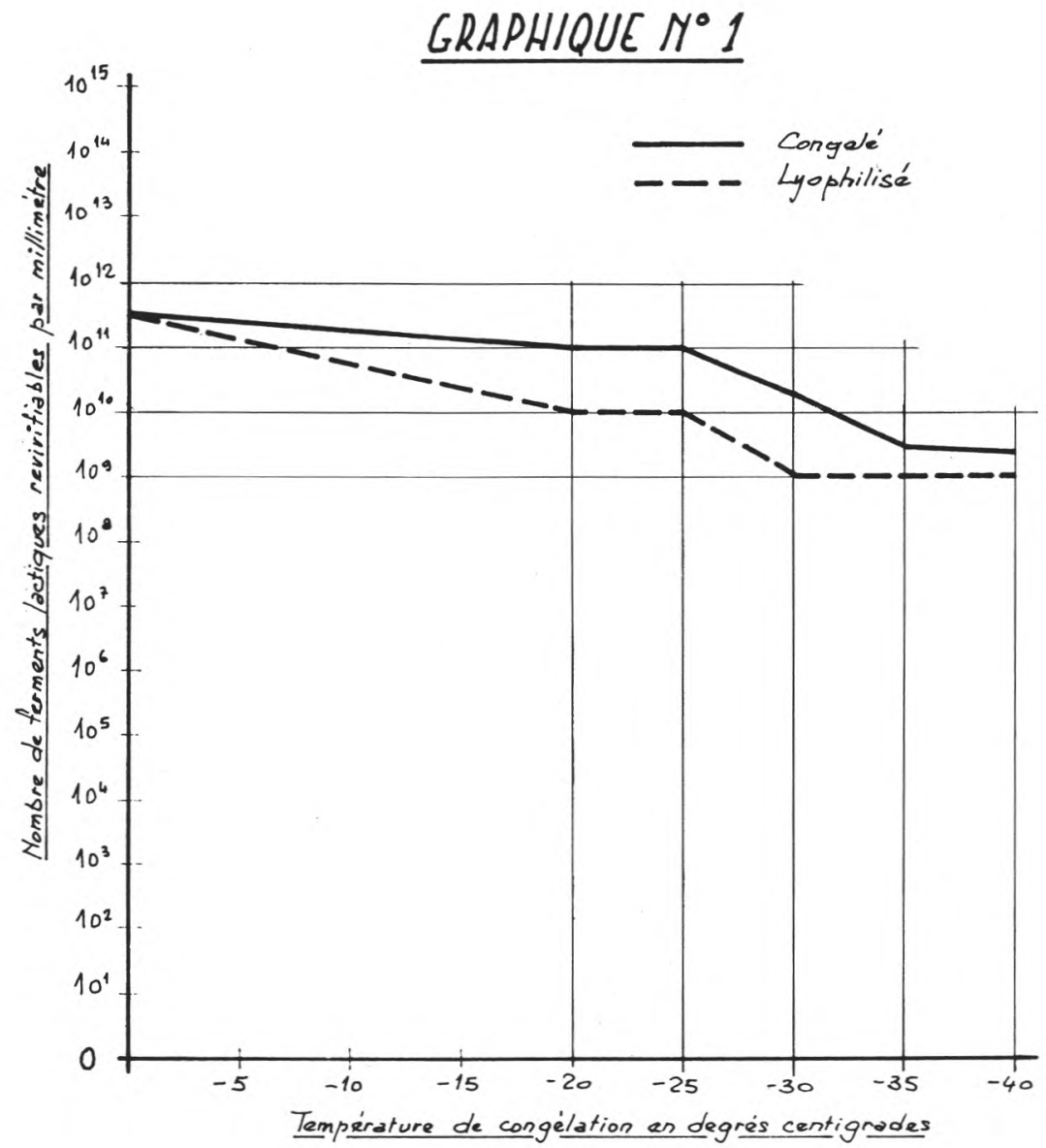

Influence des basses températures sur la survie des ferments lactiques : streptococcus thermophilus et lactobacillus bulgaricus dans le yaourt avant la phase exponentielle.

(On apprécie le seuil de sensibilité des ferments lactiques aux températures basses dans le yaourt juste avant la phase stationnaire ; la perte des ferments lactiques se stabilise à partir de $-35^{\circ} \mathrm{C}$; ce graphique a été établi sur la moyenne de 4 à 7 courbes pour chaque température de congélation, sauf pour le yaourt frais dont le taux de population a été calculé sur une moyenne de 18 échantillons). 


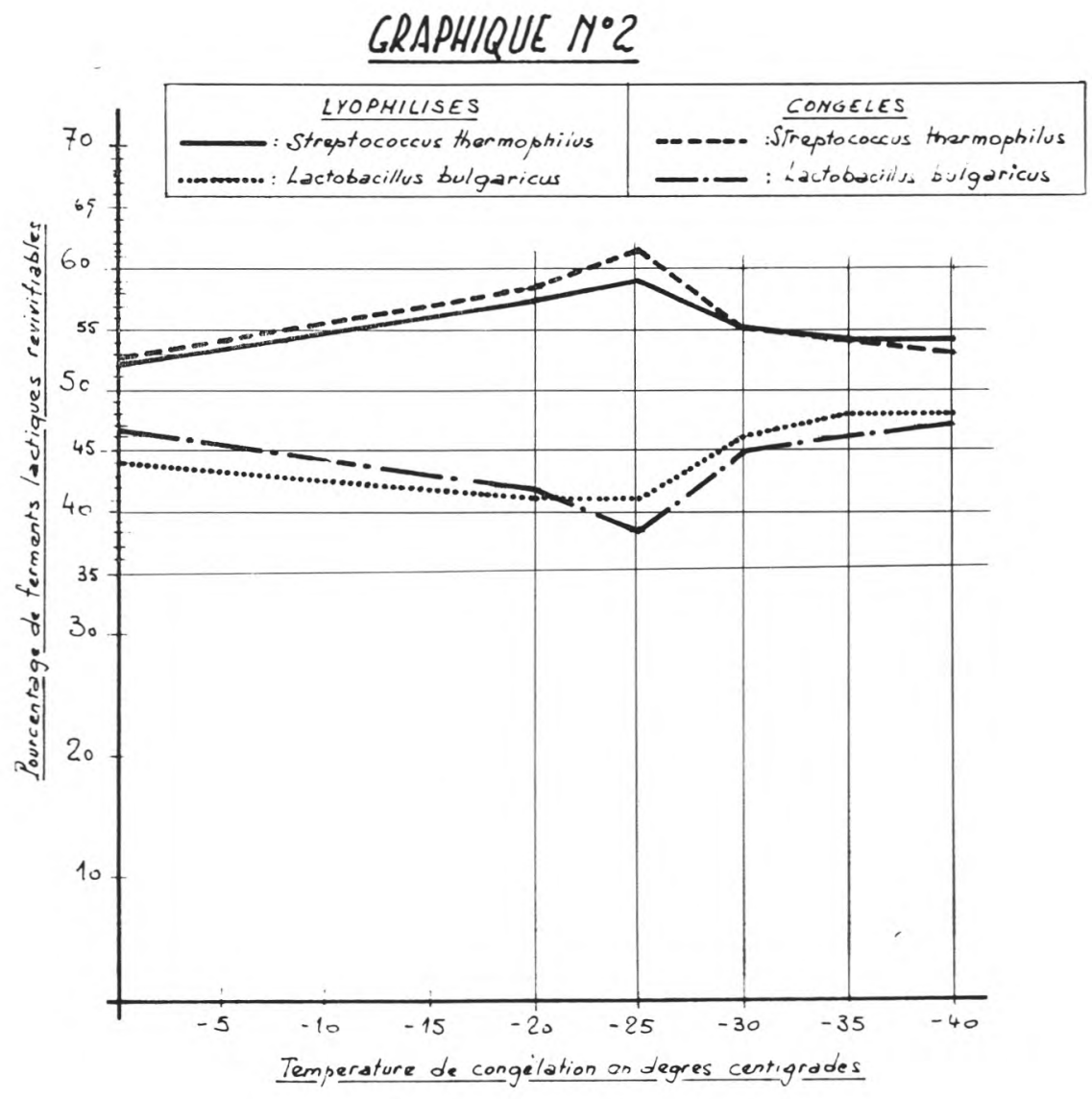

Influence des basses températures sur le pourcentage des ferments lactiques: streptococcus thermophilus, lactobacillus bulgaricus, revivifiables dans le yaourt avant la phase exponentielle.

(L'écart du pourcentage entre les ferments lactiques a tendance à s'affaiblir à partir de $-30^{\circ} \mathrm{C}$; ce graphique a été établi sur la moyenne de 4 à 7 courbes, sauf pour le yaourt frais dont le pourcentage respectif des ferments lactiques présents a été calculé sur une moyenne de 18 échantillons). 


\section{GRAPHIQUE $N^{\circ} 3$}

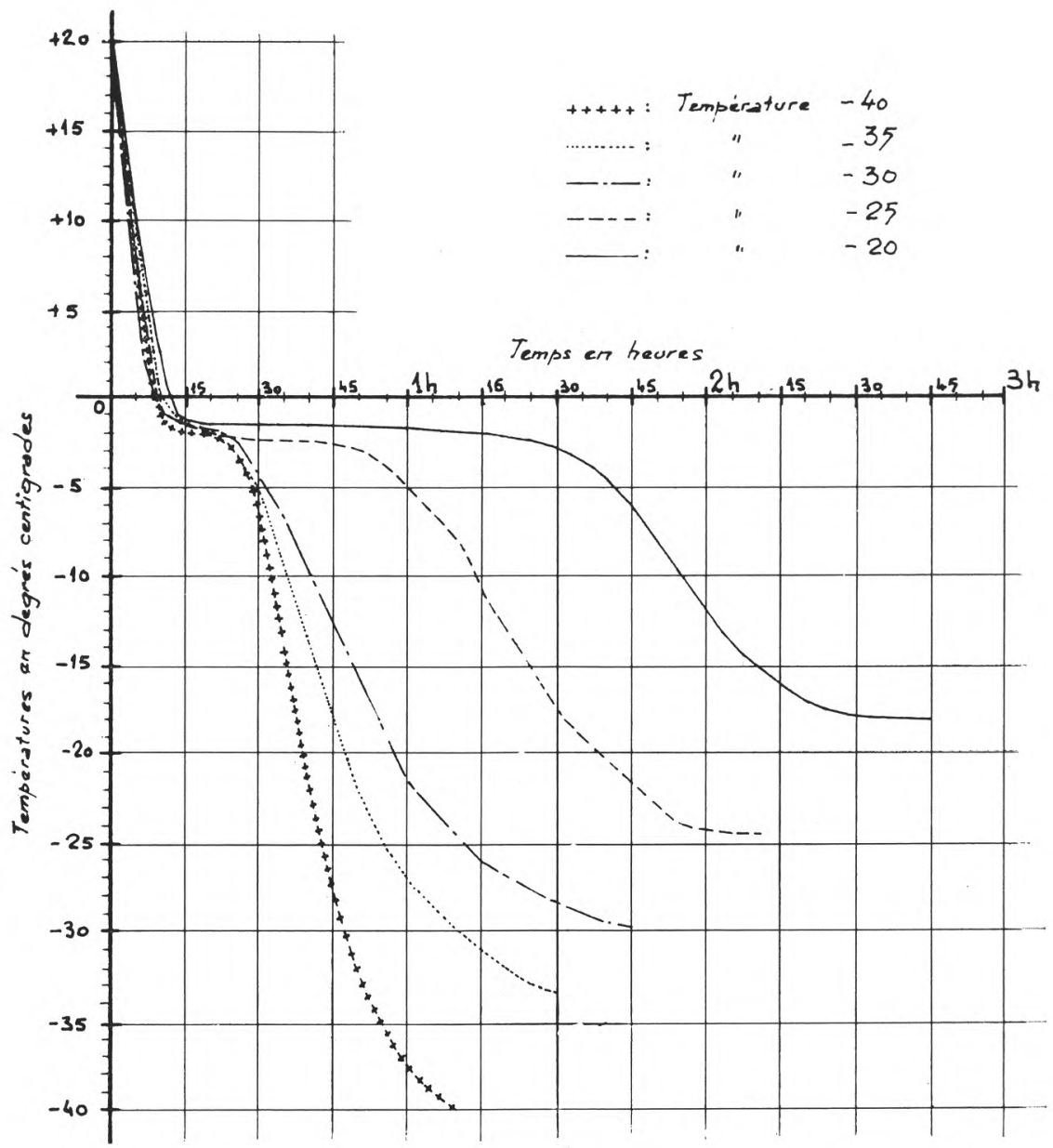

Influence des températures basses sur la durée de congélation du yaourt 


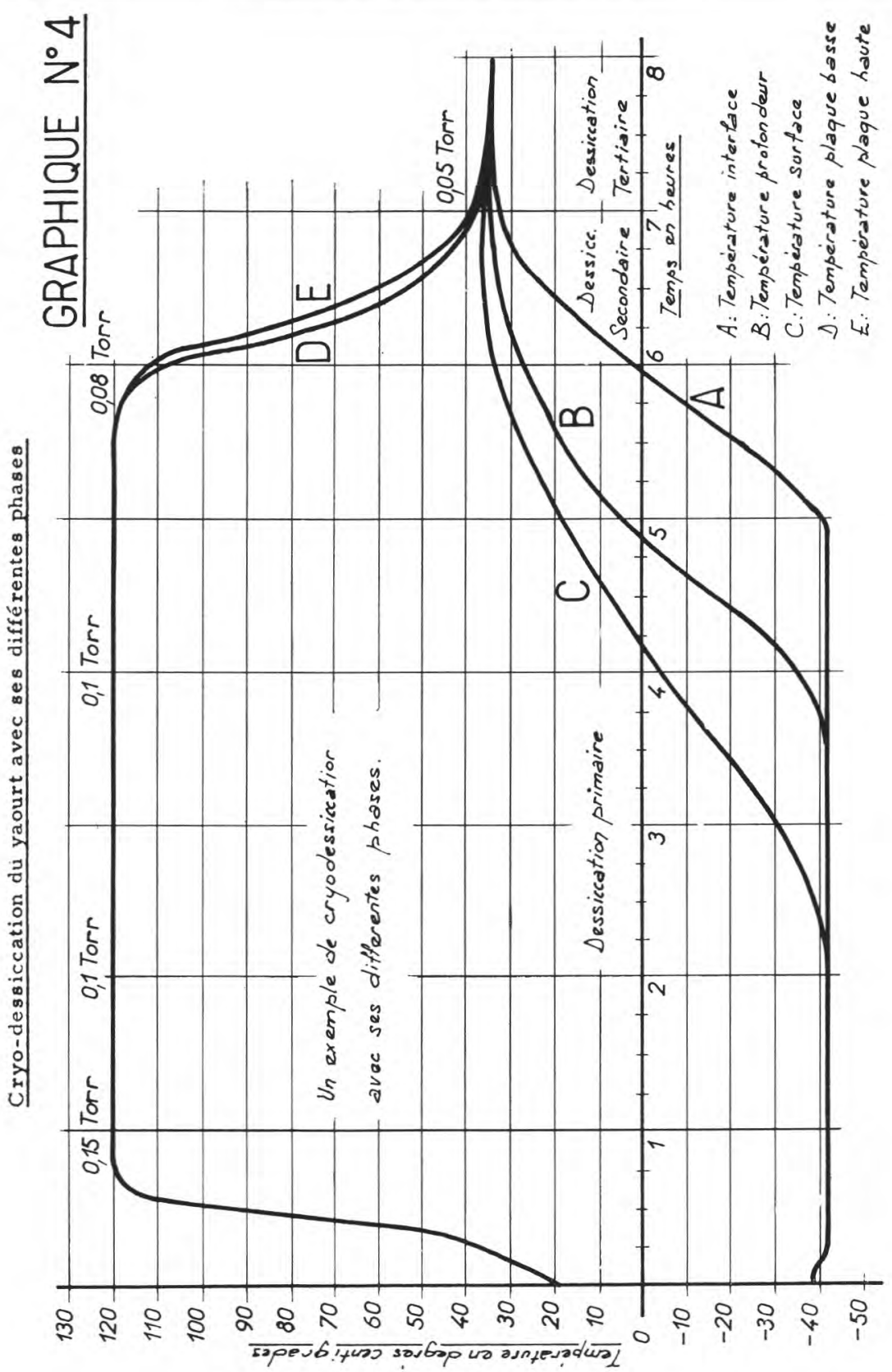

Cryo-dessiccation du yaourt et ses phases

- Dessiccation primaire : eau de sérum et eau capillaire - H.R. est supérieure à 8 p. 100 .

- Dessiccation secondaire : eau liée ou eau d'hydratation des protéines - H.R. varie entre 4 à $7 \mathrm{p} .100$.

- Dessiccation tertiaire : eau vitale des ferments lactiques et eau non cristallisable des complexes lipido-protidiques - H.R. varie entre 1 à 2 p. 100 (voire au-dessous de 1 p. 100).

Il existe une relation directe entre la pression de la vapeur et la température de chauffage pour chaque phase de la cryo-dessiccation.

(H.R. = Humidité résiduelle). 


\section{GRAPHIQUE N.5}

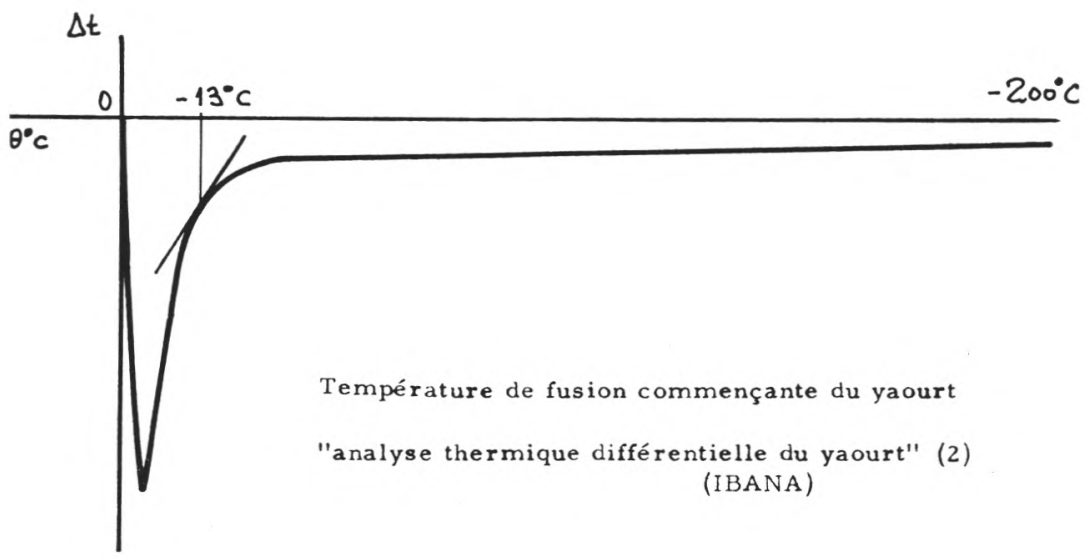

\section{GRAPHIQUE $\pi^{\circ} 6$}

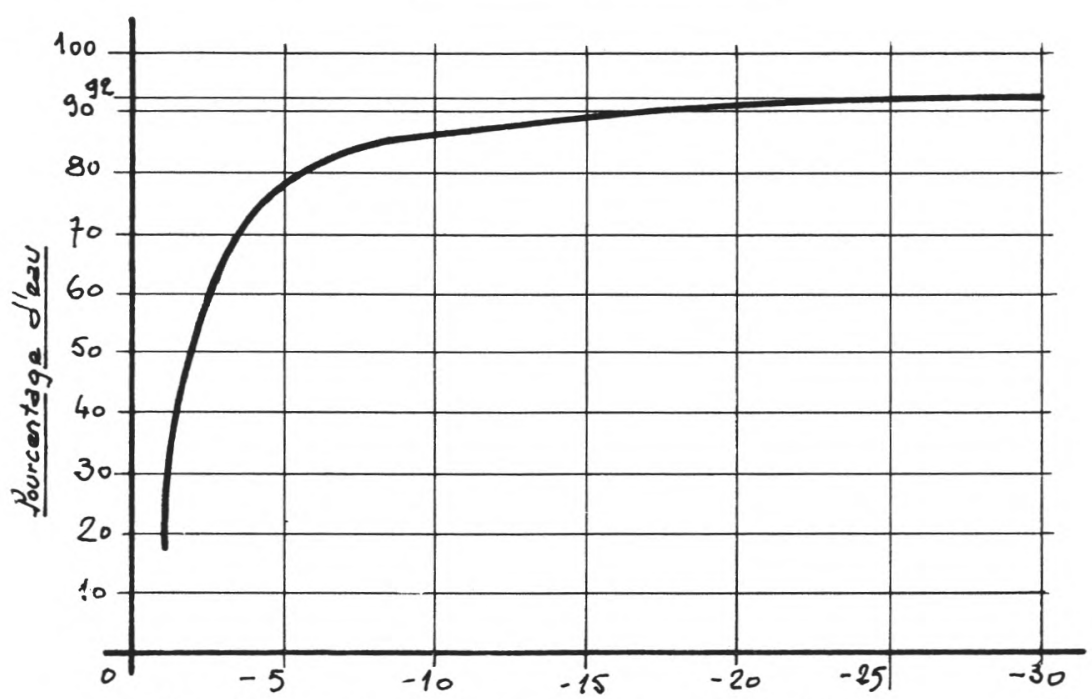

Température de congalation commen cante en $\theta^{\circ} \mathrm{C}$

Proportion d'eau transformée en glace dans un yaourt en fonction de la température (2)

(IBANA) 


\section{CONCLUSIONS}

Au cours de notre travail, nous avons recherché quelle était l'influence des basses températures sur la survie des deux souches de ferments lactiques:

- Streptococcus thermophilus,

- Lactobacillus bulgaricus.

En premier lieu, dans des conditions expérimentales déterminées, on arrive à obtenir des résultats reproductibles indiquant la sensibilité des lactobacillus bulgaricus, en fonction des températures de congélation.

En étudiant l'influence de la congélation sur la survie des deux ferments lactiques mentionnés, nous avons mis en évidence plusieurs phénomènes :

a) le froid exerce un effet énorme sur la survie des ferments lactiques, en particulier lactobacillus, le pourcentage de leur perte varie entre 7 p. 100 et 20 p. 100 suivant la température et le temps de congélation ;

b) nous avons classé quantitativement les influences des températures basses en quatre groupes:

- la température de congélation de $-20^{\circ}$ n'a pas un rôle prépondérant sur la viabilité des ferments lactiques,

- la température de congélation de $-25^{\circ}$ apparaît relativement importante sur le taux et le pourcentage des ferments lactiques revivifiables,

- la température de congélation de $-30^{\circ}$, a une nette influence sur le taux de survie des ferments lactiques étudiés mais l'écart du pourcentage de ses ferments lactiques a tendance à s'affaiblir,

- la température de congélation à partir de $-35^{\circ}$ a un rôle destructif sur le taux de survie des ferments lactiques mais l'écart $\mathrm{du}$ pourcentage des ferments lactiques diminue d'une façon sensible, de sorte qu'à la température de congélation de $-40^{\circ}$, le taux de survie des ferments lactiques est sensiblement le même qu'à $-35^{\circ}$ mais le pourcentage des ferments lactiques s'améliore de plus en plus.

En définitive, le froid n'intervient que peu sur la composition physico-chimique du yaourt lui-même, mais il a un effet nuisible sur la survie de ses ferments lactiques, en particulier les lactobacillus bulgaricus. La disproportion entre les streptocoques et les lactobacilles s'accentue au fur et à mesure que les températures baissent. Il s'ensuit que cette disproportion n'améliore pas le pourcentage respectif des ferments lactiques présents au cours de la cryo-dessiccation. 
Néanmoins, les températures très basses (graphique no 3 ) influent sur une certaine variété des ferments lactiques, sur le pourcentage et améliorent l'écart de celui-ci (le streptocoque devenant plus sensible à partir de $-25^{\circ} \mathrm{C}$ ).

Par ailleurs, nous avons constaté que la température optimale de la surface du yaourt cryo-desséché pendant la troisième phase de sublimation (dessiccation tertiaire), se situe entre $25^{\circ}$ et $35^{\circ}$ car elle permet de réduire le temps de dessiccation tertiaire. Celle-ci a un rôle efficace sur la survie des ferments lactiques cryo-desséchés (graphique $n^{\circ} 4$ ).

\section{$R$ è $\mathrm{s} u \mathrm{~m}$ é}

En définitive, le froid n'intervient que peu sur la composition physico-chimique du yaourt lui-même, mais il a un effet destructif sur la viabilité de ses ferments lactiques, en particulier, les lactobacillus bulgaricus. La disproportion entre les deux ferments lactiques s'accentue à partir de $-25^{\circ} \mathrm{C}$. Il s'ensuit que cette disproportion n'améliore pas le pourcentage respectif des ferments lactiques présents au cours de la cryo-dessiccation.

Par ailleurs, nous avons constaté que la température optimale de la surface du yaourt cryo-desséché pendant la troisième phase de sublimation (dessiccation tertiaire) se situe entre $25^{\circ} \mathrm{C}$ et $35^{\circ} \mathrm{C}$, car elle permet de réduire le temps de dessiccation tertiaire. Celleci a un rôle efficace sur la survie des ferments lactiques cryodesséchés.

\section{S u m m a ry}

Finaly, the physical and chemical compounds of yoghurt have been not affected when freezing, but the freezing temperature influences the viability of its lactic ferments, particularly "lactobacillus bulgaricus".

Disproportion between the two lactic ferments becomes important at $-25^{\circ} \mathrm{C}$. Obviously, during freeze-drying cycle, there will be no amelioration in percentage of lactic ferments.

Nevertheless, we have found, that optimal heating temperature in freeze-drying process "Third phase of sublimation" is situated between $25^{\circ} \mathrm{C}$ and $35^{\circ} \mathrm{C}$, because this temperature is important to shorten the time of "Third dessiccation" and is efficient on lactic ferments viability. 
Ce travail a été réalisé en partie avec la collaboration étroite de l'IBANA de Dijon.

Nous tenons à remercier, en particulier, M. le Professeur THIEULIN et M. le Professeur CAMUS qui nous ont donné de précieux conseils, pour la présentation de cet article.

Nos remerciements iront également à $M$. FAUCHERON, notre Directeur Général, qui nous a toujours encouragé pour la réalisation de notre travail; qu'il trouve ici, le témoignage de notre gratitude.

Nous remercions chaleureusement M. CHARON, Directeur du Centre de Recherches qui a favorisé et facilité l'aboutissement de notre travail.

\section{B i b liographie}

[1] Bergère (J.L.). - Production massive de cellules de streptocoques lactiques. La revue Le Lait, mars-avril 1968, no 473-474, p. 131.

[2] Briottet (J.M.). - Mémoire présenté à l'IBANA 1968 pour obtenir le diplôme d'Ingé. nieur IBANA.

[3] Goldblith (S.A.). - Freeze-deshydratation of foods " in researches and development in Freeze drying ». Ed. REY L. Hermann, Paris 1966.

[4] Goldblith (S.A.). - Stability of freeze dried foods " in advances in freeze - drying ". Ed. REY, L. Hermann, Paris 1966.

[5] Hoskins (J.K.). - Most probable numbers for evaluation of coli-aerogenes tests by fermentation tube methode. Public Health Wash 49 (1934), 393-405.

[6] KuprianofF. - Bound water in Food in fondamental aspect of the deshydratation of foodstuffs. Mac Millan Co (New York).

[7] Kurmann (A.). - Etudes biotechniques sur la fermentation du yoghourt doux et aromatique, La Revue Le Lait, mai-juin 1967, p. 267-287, juillet-août 1968, p. 419-431.

[8] ReY (L.). - Traité de lyophilisation. Hermann, Paris 1960.

[9] Simatos (D.). - Thèse de Doctorat ès-Sciences: l'humidité des produits lyophilisés. Faculté des Sciences, Dijon 1965.

[10] Socaltra. - Centre de Recherche, 128, bu Victor-Hugo à Clichy. - Etude sur la définition des paramètres de cryo-concentration des jus de fruits et des produits laitiers pour leur lyophilisation ultérieure. Cette étude a bénéficié de l'aide de l'action concertée de la Délégation Générale à la Recherche Scientifique. Contrat $\mathrm{n}^{\circ} 6801320002127501$. 